\title{
Low vitamin D status in relation to cardiovascular disease and mortality in Swedish women - Effect of extended follow-up
}

\section{Monica Leu Agelii ${ }^{1}$, Susanna Lehtinen-Jacks ${ }^{1,2}$, Henrik Zetterberg ${ }^{3,4,5}$, Valter Sundh ${ }^{1}$, Cecilia Björkelund $^{6}$, Lauren Lissner ${ }^{1}$}

${ }^{1}$ Section for Epidemiology and Social Medicine, Institute of Medicine, Sahlgrenska Academy, University of Gothenburg, Gothenburg, Sweden

${ }^{2}$ Faculty of Social Sciences, University of Tampere, Tampere, Finland

${ }^{3}$ Department of Psychiatry and Neurochemistry, Institute of Neuroscience a Physiology, Sahlgrenska Academy, University of Gothenburg, Gothenburg, Sweden

${ }^{4}$ Clinical Neurochemistry Laboratory, Sahlgrenska University Hospital, Mölndal, Sweden

${ }^{5}$ Department of Molecular Neuroscience, UCL Institute of Neurology, Queen Square, London, United Kingdom

${ }^{6}$ Section for Primary Health Care, Institute of Medicine, University of Gothenburg, Gothenburg, Sweden

Corresponding author: Monica Leu Agelii; email: $\underline{\text { monica.leu@gu.se }}$

\section{Background}

Vitamin D deficiency has been classically associated with impaired calcium metabolism but currently it is known that it also associates with a host of conditions, including obesity, cardiovascular disease (CVD) and dementia, and consequently with higher risk of death due to these conditions (Barnard 2010). A map of vitamin D receptors highlighted that vitamin D has a significant effects on the activity of some 200 human genes, with the vast majority of these having no obvious role in calcium management (Ramagopalan 2010). Vitamin D status is assessed by measuring circulating levels of 25-hydroxyvitamin $D$, hereafter referred to as 25D. Deficiency of 25D may impact cardiovascular risk in direct and indirect ways by activating the renin-angiotensin system and consequently increasing blood pressure, or by influencing the parathyroid hormone levels, myocardial function, inflammation and vascular calcification (Lee 2008). This is not surprising as most tissues, including the cardiac muscle, are able to metabolize 25D (Norman 2010). Moreover, low 25D status is associated with overweight and obesity (Pereira-Santos 2015, Lehtinen-Jacks 2016) which also predisposes to cardiovascular disease. A 
recent review concluded that all relevant Hill criteria for a causal link in a biological system are satisfied to indicate low 25D status as a risk factor for cardiovascular disease (Weyland 2014).

Based on a 2011 review of cardiovascular events in relation to 25D concentrations including studies with follow-up generally up to 15 years, we concluded that cardiovascular risk is particularly elevated at ranges below $25-35 \mathrm{nmol} / \mathrm{I}$ while potential benefits beyond $60 \mathrm{nmol} / \mathrm{I}$ remain unclear (Leu 2011). A meta-analysis of prospective studies, including approximately 65 000 participants, found a relative risk of 1.52 (1.30-1.77) for total CVD and 1.64 (1.27-2.10) for stroke for the lowest versus highest 25D category (Wang 2012). Another meta-analysis of 500000 participants confirmed a significant inverse relationship for all-cause mortality at concentrations less than $75 \mathrm{nmol} / \mathrm{l}$ and found no improvement or risk beyond $90 \mathrm{nmol} / \mathrm{l}$ (Garland 2014). In contrast, other studies found U- or J-shaped associations (Michaelsson 2010, Durup 2015). As the correlation between baseline 25D level and risk of disease may diminish with length of follow-up (Grant 2011, Grant 2012), widely varying follow-up times could be partly responsible for the lack of agreement between the studies.

In this article we will investigate the risk of incident total CVD, stroke and of all-cause mortality in middle-aged women that were followed for more than three decades. As this study provides one of the longest follow-ups to date on this topic, we will also examine the impact of follow-up length on the observed associations.

\section{Subjects and Methods}

The present study is based on the Population Study of Women in Gothenburg, Sweden. In 1968, women born in 1908, 1914, 1918, 1922 and 1930 were systematically sampled from the census register to yield a representative sample of 38-, 46-, 50-, 54- and 60-year-old women. Of those sampled, 1462 women (participation rate 90\%) participated in an extensive health examination in 1968-1969 (described in Bengtsson 1973). The cohort was followed prospectively with four re-examinations in 1974-1975, 1981-1982, 1992-1993 and 2000-2001. The Ethics Committee of the University of Gothenburg approved the study. All subjects gave written informed consent to participate, in accordance with the provisions of the Helsinki Declaration.

During the baseline visit, fasting blood $(120 \mathrm{ml})$ was drawn from each subject. A small quantity was used for immediate analysis and the remainder was stored at $-20^{\circ} \mathrm{C}$. The $25 \mathrm{D}$ concentrations in the stored samples were analyzed in 2013, as described elsewhere (LehtinenJacks 2016), for 1227 women ( $84 \%$ of baseline participants). The validity of 25D in this material 
has been previously investigated (Leu 2015) and these concentrations were shown to be strongly related to weight status in middle- to late-life (Lehtinen-Jacks 2016).

Baseline height was measured to the nearest $0.5 \mathrm{~cm}$ and weight was measured to the nearest $0.1 \mathrm{~kg}$ (Bengtsson 1973). Waist circumference (WC) was measured at the midway level between the lower rib margin and the iliac crest. Body mass index (BMI, $\left.\mathrm{kg} / \mathrm{m}^{2}\right)$ was calculated. In this study, BMI and WC were included as indicators of overall and central adiposity, respectively.

During the baseline clinical examination, blood pressure (BP) was measured in sitting position. Hypertension was defined as systolic BP $\geq 140 \mathrm{mmHg}$, diastolic BP $\geq 90 \mathrm{mmHg}$ or as a known history of hypertension (with or without antihypertensive medication). Information about smoking habits and physical activity during leisure time was obtained via standardized interviews (Bengtsson 1973).

Baseline education and occupational class information (OC; based on the woman's or her husband's profession, whichever was higher between them, Carlsson 1958) was obtained through questionnaires. Finally, season of 25D measurement was defined as early summer (May-June), late summer (August-October) or winter (November-April). The categories of the background factors as used in the present study are shown in Table 1.

\section{End points and follow-up time}

Morbidity data and hospital care information (diagnoses and occasions) were obtained through interviews by study physicians until 1980 and linkage with the National Patient Register from 1980 onwards. When judged necessary for adequate diagnosis the researchers examined the hospital records and death certificates and data collected at routine examinations. CVD events (myocardial infarction, heart failure, stroke and other CVD) were identified by use of ICD-8/9 codes 390-459 and ICD-10 codes I00-199. The date of the CVD event was the day the woman was admitted to the hospital with the diagnosis. The date of death was established through linkage with the national mortality register.

In the analyses of total CVD and stroke the follow-up started at the baseline examination and ended at the time of the respective event, death or on December $31^{\text {st }} 2001$, whichever occurred first. We excluded one participant with a CVD diagnosis before baseline. To examine if the effect of baseline 25D varied with the length of follow-up, we performed intermediary analyses where the follow-up was interrupted three times, each time decreasing it by five years. Consequently, the follow-up was stopped on December 31 $1^{\text {st }} 1996$, December $31^{\text {st }} 1991$ and December $31^{\text {st }}$ 1986, corresponding to 27,22 and 17 years' follow-up, respectively. The results are presented in chronological order. 
For all-cause mortality, the end of follow-up was defined as date of death or December $31^{\text {st }}$ 2006. Intermediary mortality analyses were performed where the follow-up was interrupted four times, each time decreasing the length of follow-up with five years.

\section{Statistical methods}

For statistical analyses, 25D values were dichotomized into the lowest quartile (<=51.45 nmol/L; "low 25D status") versus the upper three quartiles combined ("high 25D status"). Differences in continuous baseline factors by 25D status were tested with the t-test when the assumption of normality was fulfilled or with the Mann-Whitney test otherwise. Associations between categorical factors and 25D status were tested with the $\chi^{2}$-test.

For each outcome, the survival across 25D categories was visually inspected with unadjusted and age- and season-adjusted Kaplan Meier curves. The hazard ratio (HR) in the low 25D category was estimated from Cox proportional hazard models (high category as reference). The basic Cox models accounted for age at baseline and season of 25D measurement. The fully adjusted models accounted for known predictors of CVD morbidity or death, or factors that were significantly associated with 25D status.

Before including categorical covariates into the Cox models, we checked that they were significant according to the log-rank test and fulfilled the proportional hazard (PH) assumption (visual inspection of the $\log (-\log ($ survival)) vs log(follow-up time) plot). If this assumption did not seem fulfilled, it was further tested through the cumulative sum of martingale residuals (Lin 1993). This test was used also for continuous predictors. Those covariates that did not fulfill the $\mathrm{PH}$ assumption and were not of direct interest (age, smoking status and occupational class) were used as stratification variables.

To test the impact of follow-up length on the association between baseline 25D and outcomes, interactions between 25D status and time were introduced into the basic models. The test of such interaction assumes equality of HRs between adjacent periods and, consequently, statistical significance indicates that HR can change with time (see Appendix for details).

The fully adjusted models were built progressively, based on the entire follow-up time, by including potential confounders one at a time. For total CVD incidence and all-cause mortality, the final models included (aside from 25D status) age, season of examination, occupational class, smoking status, leisure physical activity, waist circumference, hypertension, and triglyceride level. For stroke, the selected covariates were similar except for hypertension and triglycerides. Total CVD and stroke were also adjusted for education. Collinearity between the 
variables selected in the final models was examined through the variance inflation factors and it was not detected.

Finally, we investigated if the dilution of HR towards the null (i.e. 1) across increasing periods of follow-up could be attributed to the age attained during these periods. As the Cox model did not provide enough flexibility to make this distinction, we performed sensitivity analyses using the Poisson model. A stratified analysis was run after dichotomizing the follow-up time into 1-17 vs. more than 17 years, and the age attained during these periods into 38-68 vs. 69-93 years. The models were adjusted for season of 25D measurement.

All statistical tests were 2 -sided with $\mathrm{P}<0.05$ considered statistically significant. All analyses were conducted with SAS 9.2 (SAS Institute Inc., Cary, NC, USA).

\section{Results}

At baseline, 25D status was associated with season of examination, with the lowest prevalence of low 25D from August through October (Table 1). Current smokers, women with lower occupational class, lower education, obesity and less physical activity had the highest prevalence of low 25D. Waist circumference and triglyceride levels were higher for women with a low 25D. Age, systolic and diastolic BP, and hypertension and total cholesterol were not associated with 25D status.

\section{Total CVD and stroke incidence}

Women with a low $25 \mathrm{D}$ status ( $<51 \mathrm{nmol} / \mathrm{L}$ vs $\geq 51 \mathrm{nmol} / \mathrm{L}$ ) had a significantly lower probability of remaining free of cardiovascular disease throughout the follow-up time ( $p$-value $<0.01$; Figure 1). During the first 17 years, these women had a significantly increased risk of incident CVD, $\mathrm{HR}=1.6895 \% \mathrm{Cl}(1.18 ; 2.40)$ (Table 2$)$ according to the basic Cox model. Increasing the follow-up with five years decreased the observed association to $H R=1.42(1.07 ; 1.88)$ ( $p$ for equality of HR $=0.01$ ). Additional accrual of follow-up time resulted in non-significant HRs. The increased risk at 17 years was no longer significant in the fully adjusted model.

Women in the low 25D category were more likely to experience stroke $(p<0.01$; Figure 2$)$. Their relative risk was more than 3 -fold at 17 years of follow-up in the basic model and remained unchanged in the fully adjusted model ( $H R=3.30$, Table 2$)$. In the basic model, every additional five years of follow-up diminished significantly the magnitude of risk, with HR decreasing to 1.74 at 22 years ( $p$ for equality of HRs $<0.01), 1.32$ at 27 years $(p=0.02)$ and 1.12 at 32 years $(p=0.30)$. In the fully adjusted models, the hazard ratios for periods longer than 17 years did not remain significant. 
In the sensitivity analyses, a significantly increased rate ratio of CVD was observed during the initial 17 year period in both age-groups (38-68 and 69-93 years). In contrast, after 17 years, no significant risk was found in either age-group. Regarding stroke, a highly increased risk was observed during the initial period in the 38-68-year-olds, while among older women the association could not be studied because of a limited number of events $(n=4)$. During the second period, no significant risk was found in either age-group.

\section{All-cause mortality}

Subjects with a high 25D status survived significantly longer compared to those with a low status ( $p<0.001$; Figure 3). During the initial 17 years there were 115 deaths, representing 15\% of the subjects with a low 25D status and 7\% among those with a high 25D status. Women with a low 25D had more than double the risk HR=2.26 $(1.49 ; 3.43)$ in the basic model (Table 2), which decreased to $1.66(1.38 ; 1.98)$ when the whole follow-up was included. For all examined periods, this increased risk was not explained by the additional adjustment for covariates but was of a lesser magnitude compared to the basic model. In these fully adjusted models, HR was 2.02 during the first 17 years and 1.43 at 37 years of follow-up.

\section{Discussion}

In this study, middle-aged women who were in the lowest 25D quartile had more than 3-fold increase in the risk of stroke and a 2-fold increase in the risk of death when followed for 17 years. These risks remained basically unchanged after controlling for potential confounders, but were weakened by a longer follow-up. The follow-up length had a similar attenuation effect on the risk of total CVD. Sensitivity analyses indicated that the diminishing effect of low 25D over time is mainly caused by a lengthy follow-up and not by age.

Such attenuations have also been identified by others in relation to several outcomes (Hernan 2010, Grant 2012), and their magnitudes differ depending on the disease (Grant 2011, Lim 2009). In a meta-analysis of 32000 elderly participants, HR for all-cause mortality per $20 \mathrm{nmol} / \mathrm{L}$ increase in 25D was attenuated from 0.82 at 6 years of follow-up to 0.96 after 14 years (Grant 2012). Since our cohort started in mid-life and was of a smaller size, we did not have sufficient power to estimate the impact of comparable time periods.

Different factors may explain why the magnitude of HR depends on the length of follow-up. First, over extended follow-up times HR could have a built-in selection bias as a consequence of a preferential depletion of susceptible subjects from the high-risk group (here, low 25D status) (Hernan 2010). Secondly, a single (baseline) measurement may underestimate the proportion of 
deficient subjects, as time progresses (differential misclassification), since 25D is expected to decrease by age (Gallagher 2013). Potential reasons for such decrease include reduced capacity for cutaneous production, or an increase in adipose tissue mass, leading to reduced 25D bioavailability (Pereira-Santos 2015).

We found an inverse relationship between cardiovascular risk and 25D status during the initial 22 years of follow-up, but not for longer periods. Many studies found similar associations, but the strength of these associations varies (Wang 2012, Khaw 2014, Andersson 2010). Others have not been able to confirm such relationship (Messenger 2012). Aside from differences in the mean age of the cohorts and in 25D categorization, varying follow-up lengths might explain the heterogeneity in findings.

Our results regarding stroke are of a somewhat larger magnitude compared to others (Sun 2012, Wang 2012, Andersson 2010). Generally, HR for the lowest 25D category varies between 1.5 and 2 when follow-up is limited to two decades. However the elevated risk of stroke in relation to that of a composite CVD is in line with existing knowledge that 25D shows associations with some, but not all, cardiovascular endpoints (Andersson 2010). For example, 25D status was associated with cerebrovascular death, but not coronary death (Kilkkinen 2009), suggesting different 25D mechanisms of action on these outcomes.

Regarding mortality our results are in agreement with many studies (Pilz 2009, Khaw 2014, Schöttker 2013). One such study, following 30000 middle-aged women for 20 years, found a 2fold risk among sun avoiders compared to women with the highest sun exposure (Lindqvist 2014). A recent meta-analysis confirmed a significant inverse relationship for mortality at 25D concentrations less than $75 \mathrm{nmol} / \mathrm{l}$, with double the risk in the lowest category $\leq 22 \mathrm{nmol} / \mathrm{l}$ (Garland 2014). The authors acknowledge the varying lengths of follow-up as a source of substantial heterogeneity and that the observed risk was even higher when time was limited to 7 years.

The main strengths of this study include accurate mortality and CVD information from the registers and a representative population-based cohort. We previously found that 25D levels in these samples were comparable with those expected in fresh blood regarding mean, range and winter to summer variation (Leu 2015). Further, categorizing the main exposure, instead of including the actual concentrations, should decrease somewhat the amount of misclassification bias as many participants are expected to maintain their exposure category throughout the follow-up. Another strength is that our 25D distribution-based cutoff is very close to the widely accepted level of deficiency $(50 \mathrm{nmol} / \mathrm{L})$, which enables the comparison of our results with published findings. In contrast, the main weaknesses are the small sample size and the lack of repeated 25D measurements. Consequently, we could not determine whether an augmented 
cardiovascular risk would be present also at very high 25D levels as found by others (Michaelsson 2010, Durup 2015).

In summary, low vitamin D status in mid-life increased the risk of total CVD and particularly of stroke within 17 years from the 25D assessment. Lengthier follow-up diminished this risk towards the null. Although the association with all-cause mortality was also attenuated, a heightened risk was still present after 32 years. The attenuation may be due to changes in 25D status that occurred between baseline and end of observation period. Our results, if confirmed by studies using repeated assessments of the 25D level, indicate the importance of maintaining a sufficient 25D level for CVD prevention and longevity for women throughout the late life course.

\section{References}

Andersson JL, May HT, Horne BD, et al. Relation of vitamin D deficiency to cardiovascular risk factors, disease status, and incident events in a general healthcare population. Am J Cardiol 2010; 106(7): 963-8.

Barnard K, Colon-Emeric C. Extraskeletal effects of vitamin D in older adults: cardiovascular disease, mortality, mood, and cognition. Am J Geriatr Pharmacother 2010; 8(1):4-33

Bengtsson C, Blohme G, Hallberg L, Hallstrom T, Isaksson B, Korsan-Bengtsen K, et al. The study of women in Gothenburg 1968-1969--a population study. General design, purpose and sampling results. Acta Med Scand 1973; 193: 311-318.

Carlsson G. Social mobility and class structure. Lund, Sweden:CWK Gleerup; 1958.

Durup D, Løvendahl Jørgensen H, Christensen J et al. A reversed J-shaped association between serum 25hydroxyvitamin D and cardiovascular disease mortality: the CopD study. J Clin Endocrinol Metab 2015; 100(6):2339-46.

Gallagher JC. Vitamin D and aging. Endocrinol Metab Clin North Am 2013; 42(2): 319-32.

Garland CF, Kim JJ, Mohr SB, et al. Meta-analysis of all-cause mortality according to serum 25hydroxyvitamin D. Am J Pub Health. 2014 Aug;104(8):e43-50.

Grant WB. Effect of interval between serum draw and follow-up period on relative risk of cancer incidence with respect to 25-hydroxyvitamin D level: implications for meta-analyses and setting vitamin D guidelines. Dermatoendocrinol 2011; 3(3):199-204.

Grant WB. Effect of follow-up time on the relation between prediagnostic serum 25-hydroxyvitamin D and all-cause mortality rate. Dermatoendocrinol 2012; 4(2):198-202.

Hernan MA. The hazards of hazard ratios. Epidemiology 2010; 21(1):13-15. 
Holick MF. Vitamin D deficiency. N Engl J Med, 2007; 357:266-81.

Khaw KT, Luben R, Wareham N. Serum 25-hydroxyvitamin D, mortality, and incident cardiovascular disease, respiratory disease, cancers, and fractures: a 13-y prospective population study. Am J Clic Nutr 2014; 100(5):1361-70.

Kilkkinen A, Knekt P, Aro A, et al. Vitamin D status and the risk of cardiovascular disease death. Am J Epidemiol 2009; 170(8):1032-9.

Lee JH, O'Keefe JH, Bell D et al. Vitamin D deficiency an important, common and easily treatable cardiovascular risk factor? J Am Coll Cardiol 2008; 52(24): 1949-56.

Lehtinen-Jacks S, Leu Agelii M, Hunsberger M et al. Serum 25-hydroxy vitamin D levels in middle-aged women in relation to adiposity and height trajectories over three decades. Eur J Clin Nutr 2016; doi: 10.1038/ejcn.2016.11.

Leu M, Giovannucci E. Vitamin D: epidemiology of cardiovascular risks and events. Best Pract Res Clin Endocrinol Metab 2011; 25: 633-646.

Leu M, Mehlig K, Hunsberger M et al. Quality Assessment of 25(OH)D, Insulin, Total Cholesterol, Triglycerides, and Potassium in 40-Year-Old Frozen Serum. Epidemiol Res Int 2015; Article ID 581206; doi: $10.1155 / 2015 / 581206$

Lim U, Freedman DM, Hollis BW, et al. A prospective investigation of serum 25-hydroxyvitamin D and risk of lymphoid cancers. Int J Cancer 2009; 124:979-86.

Lin DY, Wei L J, Ying Z. Checking the Cox Model with Cumulative Sums of Martingale-Based Residuals. Biometrika, 1993; 80:557-72.

Lindqvist PG, Epstein E, Landin-Olsson M, et al. Avoidance of sun exposure is a risk factor for all-cause mortality: results from the Melanoma in Southern Sweden cohort. J Intern Med 2014; 276(1): 77-86

Messenger W, Nielson CM, Li H, et al. Serum and dietary vitamin D and cardiovascular disease risk in elderly men: a prospective cohort study. Nutr Metab Cardiovasc Dis, 2012; 22(10):856-63.

Michaelsson K, Baron JA, Snellman G et al. Plasma vitamin D and mortality in older men: a communitybased prospective cohort study. Am J Clin Nutr 2010; 92: 8410848.

Norman AW, Bouillon R. Vitamin D nutritional policy needs a vision for the future. Exp Biol Med 2010; 235(9): 1034-45

Pereira-Santos M, Costa PR, Assis AM, et al. Obesity and vitamin D deficiency: a systematic review and meta-analysis. Obes Rev 2015; 16(4):341-9.

Perna L, Schöttker B, Holleczek B, Brenner H. Serum 25-hydroxyvitamin D and incidence of fatal and nonfatal cardiovascular events: a prospective study with repeated measurements. J Clin Endocrinol Metab, 2013; 98(12): 4908-15. 
Pilz S, Dobnig H, Nijpelst G et al. Vitamin D and mortality in older men and women. Clin Endoncrin, 2009; 71: 666-72.

Ramagopalan SV, Heger A, Berlanga AJ el al. A ChIP-seq-defined genome-wide map of vitamin D receptor binding: associations with disease and evolution. Genome Res, 2010; 20(10):1352-60

Schöttker B, Haug U, Schomburg L et al. Strong associations of 25-hydroxyvitamin D concentrations with all-cause, cardiovascular, cancer, and respiratory disease mortality in a large cohort study. Am J Clin Nutr, 2013; 97(4):782-93.

Sun 2012. 25-Hydroxyvitmin D levels and the risk of stroke.

Wang L, Song Y, Manson JA E et al. Circulating 25-hydroxy-vitamin D and risk of cardiovascular disease. A Meta-analysis of prospective studies. Circ Cardiovasc Qual Outcomes, 2012; 5(6):819-29.

Weyland PG, Grant WB, Howie-Esquivel J. Does sufficient evidence exist to support a causal association between vitamin D status and cardiovascular disease risk? An assessment using Hill's criteria for causality. Nutrients, 2014; 6(9): 3403-30. 
Figure 1: Age- and season-adjusted Kaplan-Meier curves for CVD incidence in the lowest 25D quartile (continuous line) and the upper three quartiles combined (dashed line).

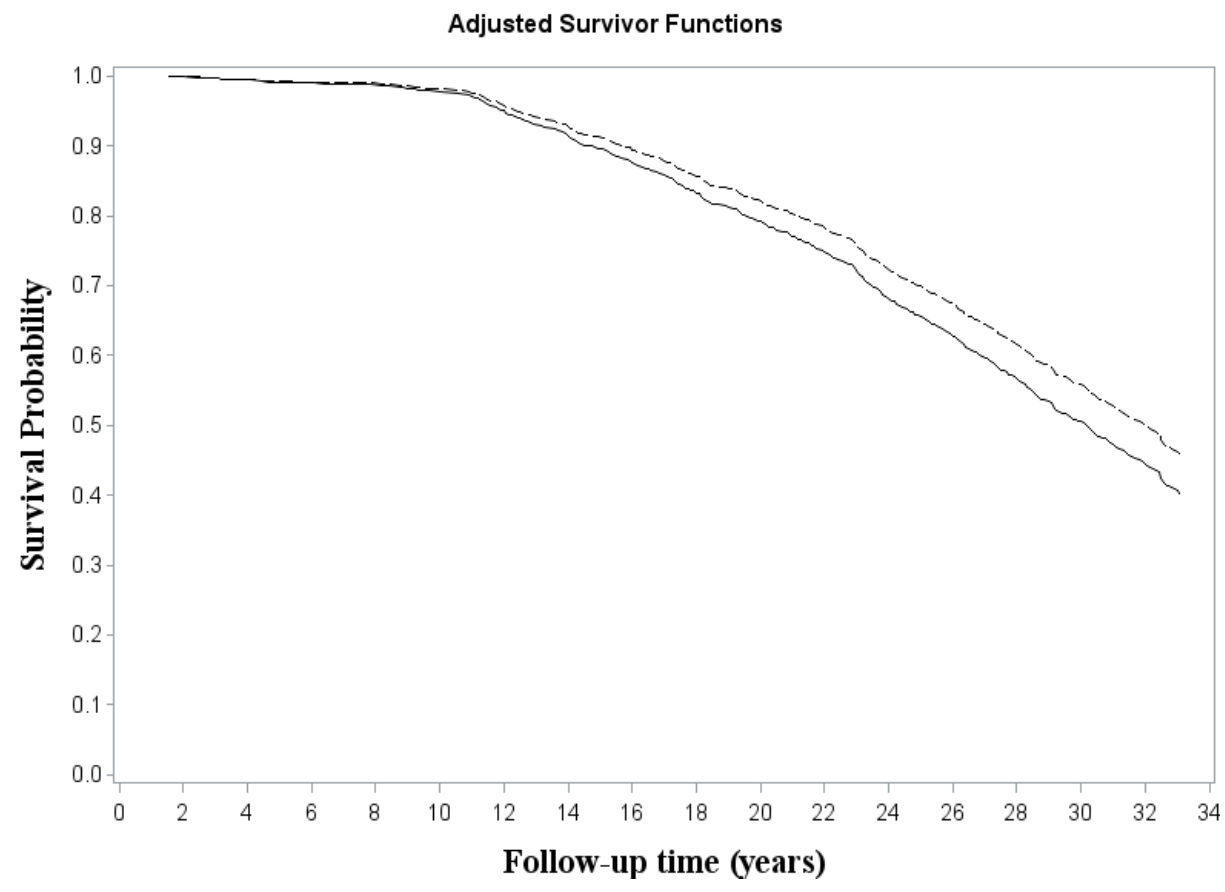

Figure 2: Age- and season-adjusted Kaplan-Meier curves for stroke incidence in the lowest 25D quartile (continuous line) and the upper three quartiles combined (dashed line).

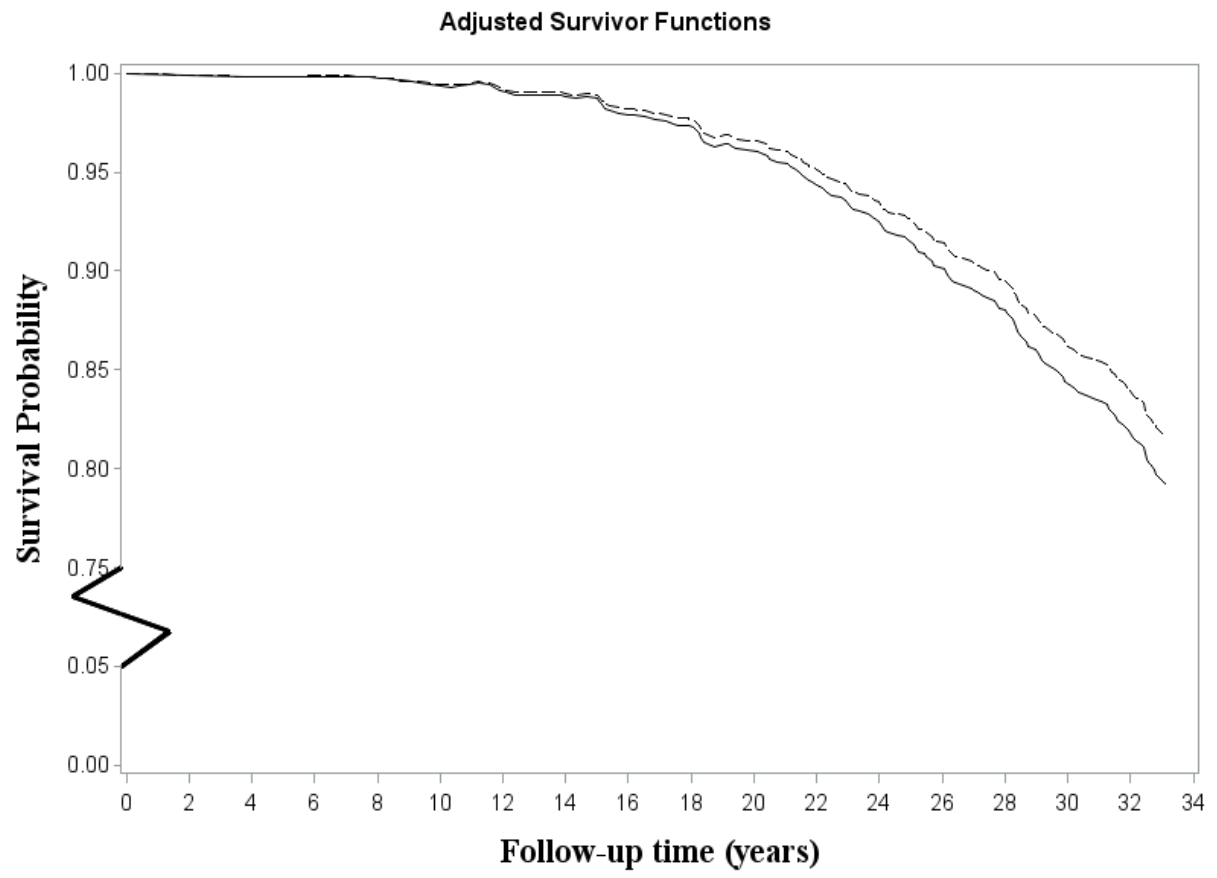


Figure 3: Age- and season-adjusted Kaplan-Meier curves for all-cause mortality in the lowest 25D quartile (continuous line) and the upper three quartiles combined (dashed line).

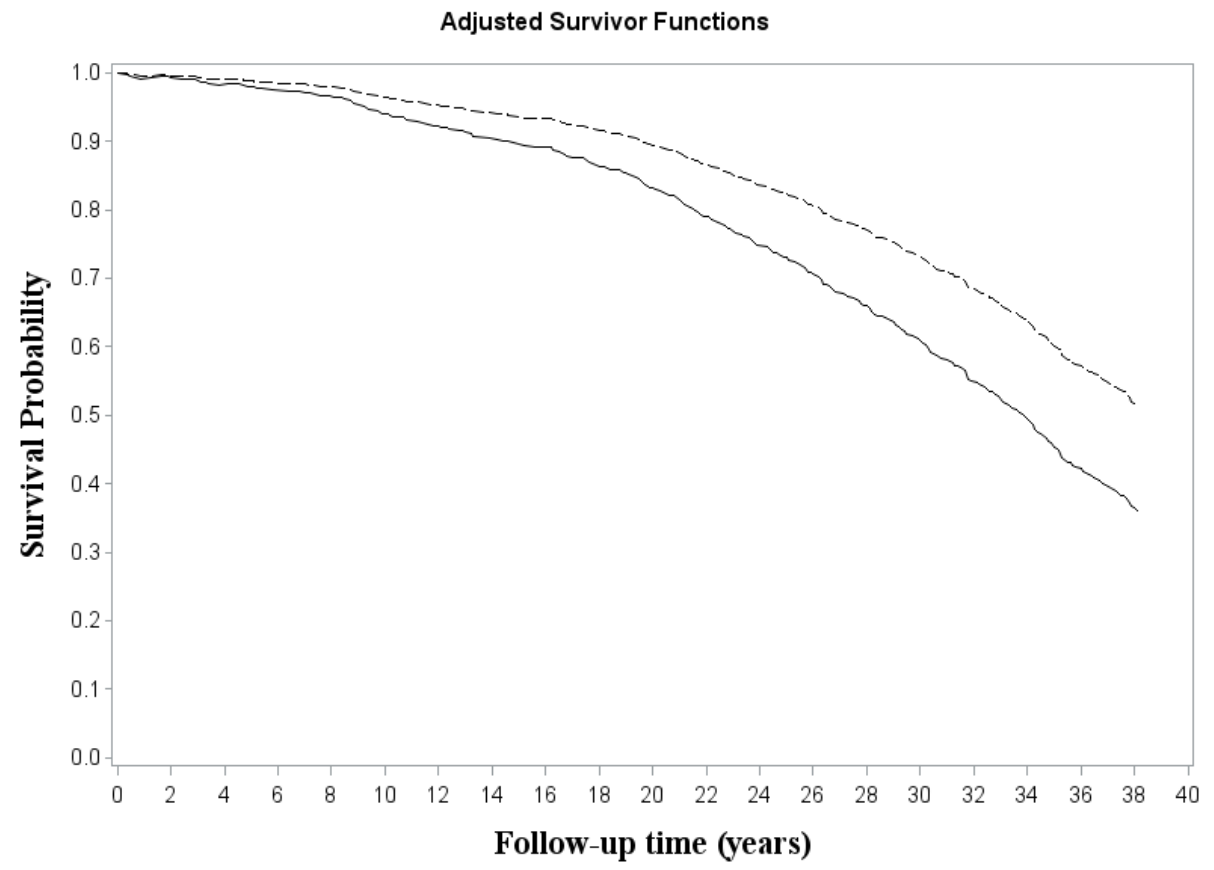


Table 1: Cross-sectional associations between vitamin D (25D) categories and baseline factors (assessed in 19681969 ) in middle-aged women, the Population Study of Women in Gothenburg, Sweden. Data are presented as prevalence percentage across 25D categories for categorical factors and mean (SE) value for continuous factors.

\begin{tabular}{|c|c|c|c|}
\hline \multirow[t]{2}{*}{ Factor } & \multicolumn{2}{|c|}{ Categories of $25 \mathrm{D}$} & \multirow[t]{2}{*}{$\mathrm{p}$ value } \\
\hline & Low $25 \mathrm{D}(<=51.45 \mathrm{nmol} / \mathrm{L})$ & High 25D (>51.45 nmol/L) & \\
\hline Age $(\mathrm{N}=1227): \%$ & & & $0.5^{\mathrm{a}}$ \\
\hline $38(n=305)$ & 26.9 & 73.1 & \\
\hline $46(n=342)$ & 25.7 & 74.3 & \\
\hline $50(n=368)$ & 25.0 & 75.0 & \\
\hline $54(n=133)$ & 18.8 & 81.2 & \\
\hline $60(n=79)$ & 25.3 & 74.7 & \\
\hline Season of examination $(\mathrm{N}=1227)$ : \% & & & $<0.001^{a}$ \\
\hline Winter (Nov-April), $\mathrm{n}=861$ & 26.7 & 73.3 & \\
\hline Early summer (May-June), $\mathrm{n}=144$ & 34.7 & 65.3 & \\
\hline Late summer (Aug-Oct), $\mathrm{n}=222$ & 12.2 & 87.8 & \\
\hline Smoking status ( $\mathrm{N}=1226): \%$ & & & $0.042^{\mathrm{a}}$ \\
\hline Never $(n=647)$ & 22.7 & 77.3 & \\
\hline Ex-smoker $(n=90)$ & 21.1 & 78.9 & \\
\hline Current smoker $(n=489)$ & 28.8 & 71.2 & \\
\hline Leisure time physical activity $(\mathrm{N}=1226$ & & & $<0.001^{a}$ \\
\hline Less than $4 \mathrm{~h} /$ week $(n=219)$ & 34.7 & 65.3 & \\
\hline At least $4 \mathrm{~h} /$ week $(n=1007)$ & 22.9 & 77.1 & \\
\hline Occupational class (OC) (N=1184): \% & & & $<0.01^{\mathrm{a}}$ \\
\hline Higher employee $(n=146)$ & 14.4 & 85.6 & \\
\hline Employee $(n=543)$ & 23.8 & 76.2 & \\
\hline Worker $(n=495)$ & 28.7 & 71.3 & \\
\hline Education $(\mathrm{N}=1222): \%$ & & & $0.02^{\mathrm{a}}$ \\
\hline Elementary school $(n=847)$ & 26.8 & 73.2 & \\
\hline Secondary school or higher $(n=375)$ & 20.3 & 79.7 & \\
\hline $\mathrm{BMI}(\mathrm{N}=1227)$ & $25.2(0.27)$ & $23.8(0.11)$ & $<0.001^{b}$ \\
\hline BMI (N=1227): \% & & & $<0.001^{\mathrm{a}}$ \\
\hline Normal $(n=817)$ & 21.2 & 78.8 & \\
\hline Overweight $(n=313)$ & 26.2 & 73.8 & \\
\hline Obese $(n=97)$ & 53.6 & 46.4 & \\
\hline Diastolic $\mathrm{BP}, \mathrm{mm} \mathrm{Hg}(\mathrm{N}=1226)$ & $85.3(0.66)$ & $84.3(0.35)$ & $0.2^{\mathrm{b}}$ \\
\hline Systolic BP, mm Hg ( $\mathrm{N}=1227)$ & $135.5(1.40)$ & $133.3(0.70)$ & $0.45^{c}$ \\
\hline Hypertension $(\mathrm{N}=1225)$ & & & $0.23^{\mathrm{a}}$ \\
\hline Yes $(n=494)$ & 26.5 & 73.5 & \\
\hline No $(n=731)$ & 23.5 & 76.5 & \\
\hline Waist circumference $(\mathrm{N}=1226)$ & $76.3(0.61)$ & $72.8(0.25)$ & $<0.001^{c}$ \\
\hline Total cholesterol, mmol/l ( $\mathrm{N}=1227)$ & $6.9(0.09)$ & $6.8(0.04)$ & $0.95^{c}$ \\
\hline Triglycerides, mmol/l (N=1226) & $1.3(0.04)$ & $1.2(0.02)$ & $0.02^{c}$ \\
\hline
\end{tabular}


Table 2: $\mathrm{HR}(95 \% \mathrm{Cl})$ of total CVD, stroke and all-cause mortality for subjects with low vitamin $\mathrm{D}(25 \mathrm{D})$ status at baseline $(\leq 51.45 \mathrm{nmol} / \mathrm{L})$ estimated from Cox proportional hazard analyses. Results are shown for increasing lengths of follow-up time.

\begin{tabular}{|c|c|c|c|c|}
\hline 17 years (ends 1986) & 22 years (ends 1991) & 27 years (ends 1996) & 32 years (ends 2001) & 37 years (ends 2006 ) \\
\hline \multicolumn{5}{|l|}{ TOTAL CVD } \\
\hline $\begin{array}{l}\text { Number }(\%) \text { of events in } \\
\text { low: high 25D category }\end{array}$ & 73 (24\%): 204 (22\%) & 102 (34\%): $336(36 \%)$ & $144(48 \%): 455(49 \%)$ & \\
\hline $1.68(1.18 ; 2.40)^{* *}$ & $1.42(1.07 ; 1.88)^{*}$ & $1.16(0.92 ; 1.47)^{(\mathrm{NS})}$ & $1.18(0.97 ; 1.44)^{(\mathrm{NS})}$ & \\
\hline $\begin{array}{l}\text { Test of equality of HR current period vs } \\
\text { previous period }{ }^{2}\end{array}$ & $p=0.01$ & $p=0.02$ & $p=0.62$ & \\
\hline Adjusted model $^{3} \quad 1.28(0.88 ; 1.88)^{(\mathrm{NS})}$ & $1.06(0.78 ; 1.42)^{(\mathrm{NS})}$ & $0.82(0.64 ; 1.05)^{(\mathrm{NS})}$ & $0.89(0.72 ; 1.09)^{(\mathrm{NS})}$ & \\
\hline \multicolumn{5}{|l|}{ STROKE } \\
\hline $\begin{array}{l}\text { Number }(\%) \text { of events in } \\
\text { low: high 25D category }\end{array}$ & $19(6 \%): 41(5 \%)$ & $27(9 \%): 78(9 \%)$ & 37 (12\%): 128 (14\%) & \\
\hline $3.54(1.55 ; 8.09) * * 5$ & $1.74(0.99 ; 3.08)^{(\mathrm{NS})}$ & $1.32(0.84 ; 2.11)^{(\mathrm{NS})}$ & $1.12(0.77 ; 1.64)^{(\mathrm{NS})}$ & \\
\hline $\begin{array}{l}\text { Test of equality of HR current period vs } \\
\text { previous period }^{2}\end{array}$ & $p<0.01$ & $p=0.02$ & $p=0.30$ & \\
\hline Adjusted model $^{4} \quad 3.30(1.37 ; 7.98)^{* * 5}$ & $1.40(0.76 ; 2.58)^{(\mathrm{NS})}$ & $0.99(0.61 ; 1.61)^{(\mathrm{NS})}$ & $0.84(0.56 ; 1.27)^{(\mathrm{NS})}$ & \\
\hline \multicolumn{5}{|l|}{ ALL-CAUSE MORTALITY } \\
\hline $\begin{array}{l}\text { Number (\%) of events in } \\
\text { low: high 25D category }\end{array}$ & $69(23 \%): 134(15 \%)$ & 102 (33\%): $211(23 \%)$ & 144 (47\%): $313(34 \%)$ & 187 (61\%): 449 (49\%) \\
\hline $2.26(1.49 ; 3.43)^{* * *}$ & $1.70(1.24 ; 2.34)^{* * *}$ & $1.65(1.28 ; 2.12)^{* * *}$ & $1.70(1.38 ; 2.09)^{* * *}$ & $1.66(1.38 ; 1.98)^{* * *}$ \\
\hline $\begin{array}{l}\text { Test of equality of HR current period vs } \\
\text { previous period }^{2}\end{array}$ & $p=0.10$ & $p=0.12$ & $p=0.77$ & $p=0.17$ \\
\hline Adjusted model $^{6} \quad 2.02(1.29 ; 3.16)^{* *}$ & $1.47(1.05 ; 2.07)^{*}$ & $1.41(1.07 ; 1.85)^{*}$ & $1.45(1.15 ; 1.81)^{* *}$ & $1.43(1.18 ; 1.73)^{* * *}$ \\
\hline \multicolumn{5}{|c|}{$\begin{array}{l}{ }^{1} \text { Model accounts for age at baseline (as a stratification variable) and season of 25D measurement. } \\
{ }^{2} \text { Comparison of HR for the current period versus HR corresponding to follow-up stopped } 5 \text { years earlier. The p-value corresponds to an } \\
\text { interaction term between 25D and a binary follow-up indicator ( } 1 \text { if the follow-up time was longer than the end of the previous follow-up period } \\
\text { and } 0 \text { otherwise). See Appendix for details. } \\
{ }^{3} \text { Model accounts for season of 25D measurement and baseline waist circumference, leisure time physical activity, hypertension, triglyceride } \\
\text { level, education, age, smoking and occupational class. Last three factors were included as stratification variables into the model. } \\
{ }^{4} \text { Model accounts for season of 25D measurement and baseline waist circumference, leisure time physical activity, education, age, smoking and } \\
\text { occupational class. Last three factors were included as stratification variables into the model. Age groups } 46 \text { and } 50 \text { years were treated as one } \\
\text { age group. }\end{array}$} \\
\hline
\end{tabular}


${ }^{5}$ Could not be adjusted for season of 25D measurement due to sparse data.

${ }^{6}$ Same adjustment factors as ${ }^{3}$ except for education

${ }^{*} p<0.05 ;{ }^{* *} p<0.01 ; * * * p<0.001$ 


\section{Appendix}

This appendix uses the comparison between the hazard ratio for the first 17 years of follow-up and the hazard ratio for the first 22 years of follow-up as an example. The same principle applies to all comparisons presented in Tables 2-4.

Denote $\mathrm{HR}_{17}$ the hazard ratio (of a given endpoint) for subjects with low 25D at baseline for the first 17 years of follow-up, $\mathrm{HR}_{22}$ the hazard ratio for the first 22 years of follow-up and $\mathrm{HR}_{17-22}$ the hazard ratio for the period 17-22 years (see Appendix figure). It is of interest to test the null hypothesis $H_{0}: H R_{17}=H R_{22}$. We show below that this hypothesis is equivalent to testing that there is no interaction between the effect of $25 \mathrm{D}$ and time i.e. that $H R_{17}$ and $H R_{17-22}$ are equal.

To obtain a p-value corresponding to the null hypothesis for the interaction $H_{0, \text { int }}: H R_{17}=$ $H R_{17-22}$ we introduce in the Cox regression model an interaction term between 25D and a dichotomous follow-up time indicator (follow-up $\leq 17$ years vs follow-up $>17$ years).

Moreover, $\mathrm{HR}_{22}$ can be expressed as a weighted average between the hazard ratio for the first 17 years of follow-up and the hazard ratio for the last 5 years of follow-up (ref Hernan 2010):

$$
H R_{22}=\frac{w_{1} H R_{17}+w_{2} H R_{17-22}}{w_{1}+w_{2}} \text { where } w_{1} \text { and } w_{2} \text { represent weights. }
$$

The null hypothesis of interest $H_{0}\left(H R_{17}=H R_{22}\right)$ above becomes

$$
\begin{gathered}
H_{0}: H R_{17}=\frac{w_{1} H R_{17}+w_{2} H R_{17-22}}{w_{1}+w_{2}} \\
<=>H_{0}: w_{1} H R_{17}+w_{2} H R_{17}=w_{1} H R_{17}+w_{2} H R_{17-22}
\end{gathered}
$$

$<=>H_{0}: H R_{17}=H R_{17-22}$, which is the same as the null hypothesis for the interaction $H_{0, \text { int }}$.

The $p$-values for the equality of HR estimates across increasing follow-up time (Tables $2-5$ ) are thus obtained from Cox regression models fitted with interaction terms between 25D and follow-up time.

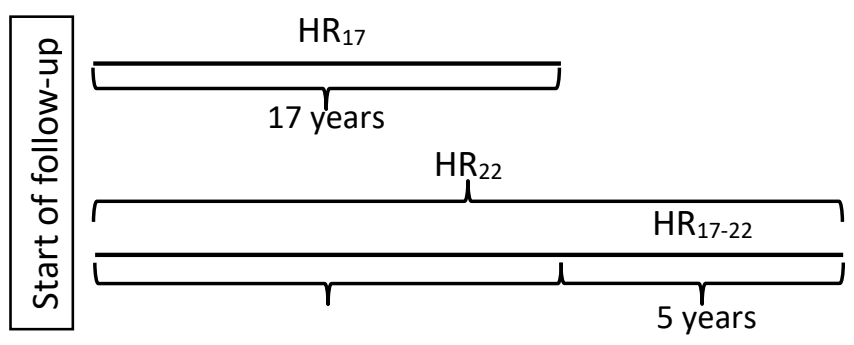

